\title{
Prognostic value of cystatin C in acute coronary syndrome
}

\author{
Mohammed Abdulwahid Shuaila, ${ }^{a}$ Hassanain M Saeed Abdulamir, ${ }^{b}$ Monem Makki Alshok ${ }^{b}$
}

\author{
aMBChB, Merjan Teaching hospital Hilla Babylon, Ministry of Health, Babylon, Iraq. \\ bDepartment of Medicine, College of Medicine, University of Babylon, Iraq. \\ Correspondence to Professor Dr Monem Alshok (email: dr_monem_alshok@yahoo.com). \\ (Submitted: 9 September 2016 - Revised version received: 2 October 2016 - Accepted: 7 October 2016 - Published online: 26 December 2016)
}

\begin{abstract}
Objective Coronary artery disease associated with an increment with a variety of markers, one of them Cystatin C, in this study we evaluate its role in a prospective study as prognostic marker, and evaluate the correlation between Cystatin C plasma level and variety of acute coronary syndrome (ACS) complications.

Methods A total 51 patients who admitted for Merjan Teaching Hospital coronary care unit whom ACS was diagnosis was made depend on history, clinical examination and investigation, and then blood sample was taken to measure plasma level and follow up for 6 months of any new events including new ischemia, rehospitalization, electrical and mechanical complication.

Results Patient who admitted with ACS with high level of cystatin $C$ associated with more mortality ( $P$ value: 0.09$)$ and more electrical complication ( $P$ value: 0.035 ) and more rehospitalization ( $P$ value: 0.01 ), but failed to show a correlation with mechanical complication. Conclusion Elevated level of Cystatin C in patient admitted to hospital with ACS associated with an increase in hospital mortality, electrical complication, and rehospitalization and lower ejection fraction than a patient with a normal Cystatin C level.

Keywords cystatin C, prognostic value, acute coronary syndrome
\end{abstract}

\section{Introduction}

Cystatin C (cys-C) is a small protein molecule (120 amino acid peptide chain, approximately $13 \mathrm{kDa}$ ) produced by virtually all nucleated cells in the human body. It belongs to the family of papain-like cysteine proteases and its main biological role is the extracellular inhibition of cathepsins. It's near constant production rate, the fact that it is freely filtered from the glomerular membrane and then completely reabsorbed without being secreted from the proximal tubular cells, made it an almost perfect candidate for estimating renal function. The strong correlation between chronic kidney disease (CKD) and cardiovascular disease (CVD) along with the growing understanding of the role of cysteinyl cathepsins in the pathophysiology of CVD inspired researchers to explore the potential association of cys-C with CVD. A high level of cystatin C in the blood corresponds to a decreased glomerular filtration rate (GFR) and hence to kidney dysfunction, Recent studies suggest that increased levels of cystatin $\mathrm{C}$ may also indicate an increased risk of heart disease, heart failure, stroke, and mortality. ${ }^{1,2,3,4}$ There is a close relationship between cystatin $\mathrm{C}$ and acute ischemic stroke, independently of conventional risk factors. ${ }^{5}$ Several studies mentioned that increased levels of cystatin $\mathrm{C}$ are found in patients with coronary artery disease. , $^{6,8,9,10}$ Cystatin $\mathrm{C}$ recently, it has been studied for its role in predicting new-onset or deteriorating cardiovascular disease. Also cystatin C may be an indicator of acute PTE (pulmonary thromboembolism) in patients with normal renal function. Also elevated serum Cystatin C levels may predict venous thrombosis beyond reflecting impaired kidney function. Cystatin $\mathrm{C}$ is known to modulate the neutrophil chemotactic activity and may inhibit prothrombotic activity of proteolytic substances secreted by activated neutrophils. Thus, it may be hypothesized that increased serum levels of cystatin $C$ represent an inadequate counterbalancing mechanism to avoid thrombosis formation. ${ }^{11-17} \mathrm{~A}$ recent study conducted by Urbonaviciene et al. demonstrated that higher serum cystatin $\mathrm{C}$ levels independently predicted 5-year all-cause and CVS mortality in symptomatic peripheral arterial disease patients with normal renal function. In accordance with Urbonaviciene et al. ${ }^{18}$ Loew and colleagues ${ }^{19}$ have reported that only high plasma cystatin $\mathrm{C}$ levels $(>1.24 \mathrm{mg} / \mathrm{l})$ were associated with risk of fatal and nonfatal cardiovascular events during the follow-up. Furthermore, in a recent meta-analysis, higher cystatin $C$ levels were strongly and independently associated with specific endpoints like stroke, myocardial infarction and heart failure. ${ }^{20,21}$

The aim of the present study was to evaluate whether the concentration of Cys C could predict the severity of coronary artery disease after myocardial infarction in patients with normal renal function estimated from the concentration of serum creatinine, and to determine the prognostic value of Cys $\mathrm{C}$ in predicting cardiovascular mortality during the follow up of patients with acute coronary syndrome (ACS).

\section{Methods}

A short-term prospective study was conducted on 51 patients with ACS admitted to Merjan teaching hospital from the first of March 2015 to first of July 2015. Patients were excluded from the study if there is any neoplasia in any organ, pregnancy, thyroid dysfunction, altered mental state, renal impairment on admission, suspected dissected aortic aneurysm, recent MI (6 month ago), patients with eGFR less than $60 \mathrm{ml} /$ $\mathrm{kg} / \mathrm{min}$, patient received steroid and any patient refuse participation. The diagnosis of ACS was established by symptoms, electrocardiography and cardiac biomarkers. Detailed history was taken from the patients, and physical examination was performed on admission. Echocardiography was done usually in the next day of admission, but bed-side echocardiography was done if there is a strong indication to assess hemodynamically unstable patients and to rule out mechanical complication in STEMI patients. Potential risk factors for ACS including hypertension (HPT), diabetes (DM), smoking, as well as age of patients, and other information were reviewed by direct questionnaire to the patients or their relatives. Risk factors were defined according to the protocol. The patients with a history of HPT or without history but compatible with JNC9 definition of hypertension $\mathrm{mm} \mathrm{Hg}$ were defined hypertensive. 
The patients who have the history of DM, being on glucose-lowering medication prior to ACS onset or without a history but who had fasting blood sugar more than $126 \mathrm{mg} / \mathrm{dl}$ or random blood sugar $>200 \mathrm{mg} / \mathrm{dl}$ on two occasions were defined diabetics. The patients were regarded as current smokers if they smoked until admission or stopped smoking within the last 3 months. GFR measured using MDRD equation (modification of diet in renal disease) as follows:

eGFR $\mathrm{ml} / \mathrm{min} .73 \mathrm{~m}^{2}=175 \times$ S.(S.Cr) $-{ }^{1} \square^{145} \times($ Age $)-$

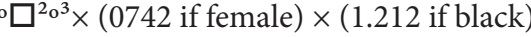

We followed the patients daily during the period of hospitalization. Clinical assessment and echocardiography was done to the patients who discharged from hospital, on average of 6 to 8 weeks after discharge. The patients were followed up by telephone or outpatient clinic visits for up to 3 months.

\section{Blood sampling and Laboratory methods}

Blood was drawn on admission at the time of inserting an intravenous line. The sample was collected into a plain tube, allowed to clot and then separate the serum by centrifuge using $\left\{\right.$ Hettich Rotofix $\left.{ }^{22}\right\}$ (A German 2005), centrifuge at a rate of $3000 \mathrm{rpm}$ for 5 minutes. $0.5-1.0 \mathrm{ml}$ of serum is stored at $2-8$ Celsius for a few days for the measurement of Cystatin C level. Serum Cystatin C is measured by using (Nephlometricimmunoterbidimetric method). The other laboratory investigation included a complete blood count, and random blood glucose, urea and creatinine. Cardiac enzymes, including troponin, if indicated we perform a thyroid function test.

\section{Statistical Analysis}

Statistical analysis was performed using statistical package for social science version 20 "SPSS 20". Categorical variable such as sex, diabetes, hypertension, smoking, occurrence of death was expressed as a percentage. The correlations between categorical variables were assessed using Chi square or Fisher exact test as appropriate. Continuous variables that were normally distributed such as the age of the patient, serum Cystatin C, duration of hospitalization were expressed as mean \pm Standard deviation. The correlation between continuous variables were assessed using annova test. $\mathrm{P}$ value of 0.05 or less considered to be significant.

\section{Results}

In this study, 51 patients were enrolled. The age ranged from 45 to 65 (mean \pm SD $52.12 \pm 8.93$ ), 32 of them were male, 19 were female, 12 patients $(23.5 \%)$ were diabetes, 15 patients $(29.4 \%)$ were hypertensive, 13 patients $(25.4 \%)$ were current smokers. Of those patients, 36 patients $(71 \%)$ were admitted with the diagnosis of STEMI, 15 patients (29\%) admitted with the diagnosis of non-STEMI/UA, among patients with STEMI 20(28\%) had anterior STEMI, 16(22\%) had Inferior STEMI. In the UA/ NSTEMI group 13(86\%) of them have ST depression/T inversion, and 2(14\%) have normal ECG findings. 33 patients (91\%) had successful reperfusion therapy, 25 patients (69\%) had PCI primary or rescue, and $8(22 \%)$ patients had thrombolysis as a sole reperfusion therapy. One patient developed acute ischemic stroke with slurred speech and dysphagia on the second day of hospitalization after primary PCI (with normal brain CT scan done immediately after the event) with the resolution of the neurological deficit during the follow up. The duration of hospitalization was $4 \pm 1.82$ days and mortality was 4 patients (7.5\%). Table 2 shows the correlation between mortality and the patients' clinical characteristics. The mean age of patients who died $(55 \pm 6.272), 3(75 \%)$ of them where males and $1(25 \%)$ where females, 2 (50\%) were hypertensive with ( $0.22 P$ value), and $3(75 \%)$ were diabetics ( $0.036 P$ value), 3 (75\%) were smokers ( $0.046 P$ value). Table 3 shows the correlation between cystatin

\begin{tabular}{ll}
\hline \multicolumn{2}{l}{ Table 1. Baseline characteristics of the patients } \\
\hline Variables & No./percent \\
\hline No. & 51 patients \\
Age & $(52.12 \pm 8.93)$ \\
Sex & 32 males/19 females \\
DM & 12 patients (23.5\%) \\
Hypertension & 15 patients (29.4\%) \\
Smoking & 13 patients (25.4\%) \\
STEMI & 36 patients $70.5 \%)$ \\
NonSTEMINUA & 15 patients (28.3\%) \\
Reperfusion in STEMI & 33 patients (62.2\%) \\
Thorombolysis & 8 patients (15\%) \\
PCI & 25 patients (47.1\%) \\
ECG findings & \\
Anterior STEMI & $20(39.2 \%)$ \\
Inferior STEMls & $16(30.1 \%)$ \\
ST depression\T inversion & $13(24.5 \%)$ \\
Normal ECG & $2(3.7 \%)$ \\
Mortality & 4 patients (7.5\%) \\
S. cystatin & \\
Duration of hospitalization & $(4$ days \pm 1.82$)$ \\
\hline
\end{tabular}

\begin{tabular}{|c|c|c|c|}
\hline \multirow{2}{*}{ Variables } & \multicolumn{2}{|c|}{ In hospital mortality } & \multirow{2}{*}{$P$ value } \\
\hline & Yes (4) & № (49) & \\
\hline Age & $55 \pm 6.272$ & $51.87 \pm 9.138$ & NS \\
\hline Sex & $\begin{array}{c}3(5.6 \%) \\
\text { males } 1(1.8 \%)\end{array}$ & & 0.22 \\
\hline Hypertension & $2(3.7 \%)$ & $2(3.7 \%)$ & 0.22 \\
\hline DM & $3(5.6 \%)$ & $1(1.8 \%)$ & 0.036 \\
\hline Smoking & $3(5.6 \%)$ & $1(1.8 \%)$ & 0.046 \\
\hline
\end{tabular}

\begin{tabular}{|c|c|c|c|c|c|}
\hline variables & & No. & $\begin{array}{c}\text { Mean } \\
\text { Cystatin C }\end{array}$ & $\pm S D$ & $P$ values \\
\hline \multirow[t]{2}{*}{ Mortality } & died & 4 & 0.8538 & 0.48531 & 0.009 \\
\hline & no & 47 & 1.5250 & 0.30838 & \\
\hline \multirow{2}{*}{$\begin{array}{l}\text { Electrical } \\
\text { complications }\end{array}$} & yes & 4 & 1.4150 & 0.61016 & 0.035 \\
\hline & no & 47 & 0.8632 & 0.47869 & \\
\hline \multirow[t]{2}{*}{ Rehospitalization } & yes & 9 & 1.3833 & 0.40159 & 0.01 \\
\hline & No & 42 & 0.8043 & 0.46864 & \\
\hline \multirow{2}{*}{$\begin{array}{l}\text { Mechanical } \\
\text { complications }\end{array}$} & yes & 1 & 1.7000 & & 0.1 \\
\hline & no & 50 & 0.8906 & 0.49770 & \\
\hline
\end{tabular}

J Contemp Med Sci| Vol. 2, No. 8, Autumn 2016: 119-122 
level and mortality, re hospitalization, electrical and mechanical complications. In patients who died during the hospital course were $1.5250 \pm 0.30838$, while the mean cystatin c level in patients who were discharged alive from the hospital were between 0.8538 and 0.8531 . The correlation was statistically significant $(P$ value 0.009). 4 patients (19\%) developed electrical complications in hospital. Two patients developed primary ventricular fibrillation (VF) were successfully resuscitated, one patient developed atrial fibrillation in which rate controls done with intravenous amiodarone infusion, and one patient developed sustained ventricular tachycardia (VT) was successfully resuscitated with mean cystatin C $(1.4150 \pm 61016)$ SD developed electrical ( $P$ value 0.035 significant). Also our results showed correlation between Cystatin C level and ejection fraction (EF) using Spearman's correlation coefficient, which showed a negative correlation coefficient $(-154)$. Nine patients $(17.6 \%)$ readmitted to hospital, of those patient 4 patients admitted with decompensated heart failure, 2 patients admitted due to post MI angina, 2 patients due to acute pulmonary edema, and 1 patient due to VSR. With mean 1.3833 and std. deviation 0.40159 . VSR in which 1 patient $(1.9 \%)$ developed VSR. This patient developed clinical deterioration with hemodynamic instability and developed a new murmur with auscultation, ECHO approves the presence of acquired VSD and the patient referred for urgent surgical intervention ( $P$ value 0.1 non-significant)

\section{Discussion}

In this study, we have found that an increase in Cystatin-C level is associated with an increase in hospital mortality. This result has agreed with Osama Tayeh et al. ${ }^{23}$ Their study was a non-randomised controlled trial took place in Egypt, prospectively has conducted on 75 patients with (ACS) and also an equal number of controls. Patients who have included in this study are presented with ACS and evaluated the prognostic value of it as a predictor for the major acute coronary event). Our concern study has shown that group with a high Cystatin C level is associated with significant in hospital mortality $(P$ value 0.025$)$, and also it is going with Leila Abid et al., ${ }^{24}$ Which showed ( $P$ value 0.01 clinically significant). And another study where Cystatin C was used as a prognostic biomarker in STEMI and Shlipak et al. ${ }^{25}$ Eriksson et al. have asserted that an increase in cholesterol or LDL-cholesterol levels are considered as risk factors of IHD, but CYS-C may reflect precisely the presence or absence of CAD ${ }^{26}$ We found in this study that diabetes and smoking are associated with increasing mortality that agrees with Osama Tayehetal. and presenting significant correlation with smoking and disagree with Leila Abidetal. (non-significant). While in a multivariate regression analysis between the prevalence of high CYS-C and other common traditional risk factors, including: hypertension, diabetes, and smoking if all risk factors are present, the patient had a high CYS-C level Meanwhile hypertensive was clinical regarded as nonsignificant ( $P$ value 0.22$)$. The reasonable explanation for our results is that serum cystatin $C$ has regarded as the most sensitive marker of early renal dysfunction, due to that it may be involved in the process of coronary heart disease. Despite the uncertainty of the exact mechanisms underlying the predictive role of cystatin $\mathrm{C}$ in $\mathrm{CHD}$, evidence suggests that elevated serum cystatin $\mathrm{C}$ is associated with worse prognosis in patients with CHD. A study by Zethelius et al. have declared whether a combination of biomarkers, including $\mathrm{N}$-terminal pro-brain natriuretic peptide, cystatin $\mathrm{C}$, troponin, and hs-CRP, have improved patient stratification of risk compared with established cardiovascular risk factors. Those researchers have discovered that adding cystatin $\mathrm{C}$ to the system significantly will improve predictive efficacy. ${ }^{27}$ Also, we found that higher patient with higher level of Cystatin $\mathrm{C}$ levels have associated with increased incidence in hospital electrical complications ( $P$ value 0.035$)$, and this agrees with Osama Tayah et al. who have confirmed that higher level of Cystatin $C$ is associated with a higher chance of electrical complications $(P$ value 0.029). And we also found that high level of Cystatin $\mathrm{C}$ associated with higher rehospitalization due to various causes (decompensated heart failure, post MI angina, pulmonary edema, etc.) that agreed with Ichimoto et al. ${ }^{28}$ whom investigated the Cystatin level of 71 patients with STEMI, had also suggested the prognostic value of $\mathrm{Cys} \mathrm{C}$, high concentrations of this marker were associated with greater frequency of rehospitalization and acute heart failure episodes. Association of Cystatin $\mathrm{C}$ with greater mortality rate during follow-up. Kilic et al. ${ }^{29}$ have investigated 160 patients hospitalized with ACS and demonstrated that the admission of the serum CYSC level was significantly associated with future cardiovascular complications and rehospitalization and mortality during 12 months of follow-up. It also agrees with Osama Tayeh and Axel Akerblom et al. ${ }^{30}$ Higher level of Cystatin C is associated with lower ejection fraction (Spearman's correlation coefficient which showed a negative correlation coefficient $(-0.154)$. This also has agreed with Moran et al. who have concluded that CYS-C can be a marker of heart failure, and its combination with $\mathrm{N}$-terminal pro-B-type natriuretic peptide $(\mathrm{Nt}-$ Pro-BNP) considered to be as good marker than CYS-C alone for predicting cardiovascular mortality, especially in elderly patients with heart failure. ${ }^{31}$ Our study agrees with Garcia Acuna et al. who indicated that an elevated serum CYS-C level will predict the development of myocardial infarction, heart failure and cardiovascular death in 203 patients hospitalized with high-risk ACS, independent of other classical risk factors either in-hospital or during a 6-month follow-up period..$^{32}$ The results of our study also have agreed with Silva et al. ${ }^{33}$ who have suggested that patients admitted for ST elevation myocardial infarction and who presented elevated Cys C levels $(P$ $0.84 \mathrm{mg} / \mathrm{L}$ ) on admission, had greater risk of progression to cardiogenic shock or death during hospitalization. In this same study, only Cys C levels P $0.84 \mathrm{mg} / \mathrm{L}$ and impaired LVEF $<40 \%$ were the predictors of the risk of death during the follow-up. And also agreed with Ichimoto et al. and Osama et al. Mechanical complications have shown non-significant results ( $P$ value 0.1 ), which had disagreed with Osama Tayeh et al. ( $P$ value 0.002 ), and this might be due to small a number of data that we have used in our study.

\section{Conclusion}

Elevated level of Cystatin C in patients admitted to the hospital with ACS associated with an increase in hospital mortality, electrical complication, and re hospitalization and lower ejection fraction than a patient with a normal Cystatin $\mathrm{C}$ level. We recommend measuring the Cystain $\mathrm{C}$ level with early hours of admission for any patient of ACS for risk stratification and early possible intervention.

\section{Conflict of Interest}

None. 


\section{References}

1. Angelidis C, Deftereos S, Giannopoulos G, Anatoliotakis N, Bouras G, Hatzis $\mathrm{G}$, et al. Cystatin C: an emerging biomarker in CV disease. Curr Top Med Chem Athens Greece. 2013;13:164-179.

2. Shlipak MG, et al. Cystatin C versus Creatinine in Determining Risk Based on Kidney Function. N Engl J Med 2013;369:932-943. Online at: http:// www.nejm.org/doi/full/10.1056/NEJMoa1214234 through http://www. nejm.org.

3. Henry's Clinical Diagnosis and Management by Laboratory Methods. 21st ed. McPherson R, Pincus M, eds. Philadelphia, PA: Saunders Elsevier: 2007, 153-154

4. Inker LA, Schmid CH, Tighiouart H, Eckfeldt JH, Feldman HI, Greene T, et al. Estimating Glomerular Filtration Rate from Serum Creatinine and Cystatin C. N Engl J Med. 2012;367:20-29.

5. Huang GX, Ji XM, Ding YC, Huang HY. Association between serum cystatin C levels and the severity or potential risk factors of acute ischemic stroke. Neurol Res. 2016;38(6):518-523.

6. Dupont M, Wu Y, Hazen SL, Tang WH. Cystatin C Identifies patients with stable chronic heart failure at increased risk for adverse cardiovascular events. Circ Heart Fail. 2012;5:602-609.

7. Ix JH, Shlipak MG, Chertow GM, Ali S, Schiller NB, Whooley MA, Cystatin C. left ventricular hypertrophy, and diastolic dysfunction: data from the Heart and Soul Study. J Card Fail. 2006;12:601-607.

8. Ix JH, Shlipak MG, Liu HH, Schiller NB, Whooley MA. Association between renal insufficiency and inducible ischemia in patients with coronary artery disease: the heart and soul study. J Am Soc Nephrol. 2003;14:3233.

9. Ni L, Lü J, Bo Hou L, Tao Yan J, Fan Q, Hui R, et al. Cystatin C, associated with hemorrhagic and ischemic stroke, is a strong predictor of the risk of cardiovascular events and death in Chinese. Stroke. 2007;38:3287-3288.

10. Windhausen F, Hirsch A, Fischer J, van der Zee PM, Sanders GT, van Straalen $J$, et al. Cystatin C for enhancement of risk stratification in non-ST elevation acute coronary syndrome patients with an increased troponin T. Clin Chem. 2009;55:1118-1125.

11. Ellen E. Brodin, Sigrid K. Brækkan, Anders Vik, Jan Brox, John-Bjarne Hansen Haematologica. 2012;97:1008-1013.

12. Massberg S, Grahl L, von Bruehl ML, Manukyan D, Pfeiler S, Goosmann C, et al. Reciprocal coupling of coagulation and innate immunity via neutrophil serine proteases. Nat Med. 2010;16:887-896.

13. Leung-Tack J, Tavera C, Martinez J, Colle A. Neutrophil chemotactic activity is modulated by human cystatin C, an inhibitor of cysteine proteases. Inflammation. 1990;14:247-258.

14. Helmersson-Karlqvist J, Johan Ärnlöv Axel C. Carlsson, Härmä J, Larsson A. Increased urinary cystatin $C$ indicated higher risk of cardiovascular death in a community cohort. Atherosclerosis. 2014;234:108-113.

15. Johannes A. Cystatin C and the relation to cardiovascular disease: studies on the relative importance of genetic and environmental factors. doctoral theses department of medicine Karolinska University Etssjukhuset, Solna, 2015.

16. Dardashti A, Nozohoor S, Algotsson L, Ederoth P, Bjursten $\mathrm{H}$. The predictive value of s-cystatin C for mortality after coronary artery bypass surgery. J Thorac Cardiovasc Surg. 2016;152:139-146.

17. Dent TH. Predicting the risk of coronary heart disease. II. The role of novel molecular biomarkers and genetics in estimating risk, and the future of risk prediction. Atherosclerosis. 2010;213:352-362.
18. Urbonaviciene G, Shi GP, Urbonavicius S, Henneberg EW, Lindholt JS. Higher cystatin C level predicts long-term mortality in patients with peripheral arterial disease. Atherosclerosis. 2011;216:440-445.

19. Loew M, Hoffmann MM, Koenig W, Brenner H, Rothenbacher D. Genotype andplasma concentration of cystatin C in patients with coronary heart disease and risk for secondary cardiovascular events. Arterioscler Thromb Vasc Biol. 2005;25:1470-1474.

20. Lee M, Saver JL, Huang WH, Chow J, Chang KH, Ovbiagele B. Impact of elevatedcystatin C level on cardiovascular disease risk in predominantly high cardio-vascular risk populations: a meta-analysis. Circ Cardiovasc Qual Outcomes. 2010;3:675-683.

21. Salgado JV, Souza FL, Salgado BJ. How to understand the association between cystatin C levels and cardiovascular disease: Imbalance, counterbalance, or consequence? J Cardiol. 2013;62:331-335.

22. Servais A, Giral P, Bernard M, Bruckert E, Deray G. Isnard, Bagnis, C. Is serum cystatin-C a reliable marker for metabolic syndrome? Am J Med. 2008;121:426-432.

23. Tayeh O, Rizk A, Mowafy A, Salah S, Gabr K. Cystatin-C as a predictor for major adverse cardiac events in patients with acute coronary syndrome. Egyptian Heart J. 2012;64:87-95.

24. Abid L, Charfeddine S, Kammoun S, Turki M, Ayedi F. Cystatin C. A prognostic marker after myocardial infarction in patients without chronic kidney disease. J Saudi Heart Assoc. 2016;28:144-151.

25. Shlipak MG, Katz R, Sarnak MJ, Fried LF, Newman AB, Stehman-Breen C, et al. Cystatin $C$ and prognosis for cardiovascular and kidney outcomes in elderly persons without chronic kidney disease. Ann Intern Med. 2006;145:237-246.

26. Eriksson P, Jones KG, Brown LC, Greenhalgh RM, Hamsten A, Powell JT. Genetic approach to the role of cysteine proteases in the expansion of abdominal aortic aneurysms. Br J Surg. 2004;91:86-89.

27. Zethelius B, Berglund L, Sundström J, et al. Use of multiple biomarkers to improve the prediction of death from cardiovascular causes. N Engl J Med. 2008;358:2107-2116.

28. Ichimoto E, Jo K, Kobayashi Y, Inoue T, Nakamura Y, Kuroda N, et al. Prognostic significance of cystatin C in patients with ST-elevation myocardial infarction. Circ J. 2009;73:1669-1673.

29. Teoman K, Gokhan O, Ertan U, Zeki Y, Ulas S, Ulas B, et al. Comparison of the long-term prognostic value of Cystatin $C$ to other indicators of renal function, markers of inflammation and systolic dysfunction among patients with acute coronary syndrome. J Atheroscler. 2009;207: 552-558.

30. Ix JH, Shlipak MG, Chertow GM, Whooley MA. Association of cystatin C with mortality, cardiovascular events, and incident heart failure among persons with coronary heart disease: data from the Heart and Soul Study. Circulation 2007;115:173-179.

31. Moran A, Katz R, Smith NL. Cystatin C concentration as a predictor of systolic and diastolic heart failure. J Card Fail. 2008;14:19-26.

32. Jose GAM, Eva GB, Lilian GD, Carlos PG, Rafael VP, Ana LL. Prevalence of disease of the large arteries in an elderly Belgian population: Relationship with some metabolic factors. Acta Cardiol. 1984;39: 365-372.

33. Silva D, Cortez-Dias N, Jorge C, Marques JS, CarrilhoFerreira P, Magalhães $A$, et al. Cystatin C as prognostic biomarker in ST-segment elevation acute myocardial infarction. Am J Cardiol. 2012;109:1431-1438. 